\title{
Prediction of Respiratory Failure and Mortality in COVID-19 Patients Using Long Pentraxin PTX3
}

\author{
Cecilie Bo Hansen ${ }^{\mathrm{a}}$ Håkon Sandholdt ${ }^{\mathrm{b}}$ Maria Elizabeth Engel Møller ${ }^{\mathrm{b}}$ \\ Laura Pérez-Alós $^{a}$ Lise Pedersen $^{c}$ Simone Bastrup Israelsen ${ }^{b}$ Peter Garred ${ }^{a} d$ \\ Thomas Benfield ${ }^{\text {b, } d}$ \\ aLaboratory of Molecular Medicine, Department of Clinical Immunology, Section 7631, Rigshospitalet University \\ Hospital, Copenhagen, Denmark; ${ }^{b}$ Department of Infectious Diseases, Copenhagen University Hospital - Amager \\ and Hvidovre, Hvidovre, Denmark; ' Department of Clinical Biochemistry, Holbaek Hospital, Holbaek, Denmark; \\ ${ }^{\mathrm{d}}$ Department of Clinical Medicine, Faculty of Health and Medical Sciences, University of Copenhagen, Copenhagen, \\ Denmark
}

\author{
Keywords \\ Long pentraxin-3 $\cdot$ PTX3 $\cdot$ COVID-19 $\cdot$ Inflammation $\cdot$ Critical \\ illness
}

\begin{abstract}
The course of COVID-19 is unpredictable, ranging from asymptomatic to respiratory failure and death. Prognostic biomarkers are urgently needed. We hypothesized that long pentraxin PTX3 could be a valuable plasma biomarker due to its essential role in inflammatory processes. In a prospective hospitalized COVID-19 derivation cohort $(n=126)$ during the spring of 2020, we measured PTX3 within 4 days of admission. The predictive value of mechanical ventilation (MV) and 30-day mortality compared with clinical parameters and other markers of inflammation were assessed by logistic regression analysis and expressed as odds ratio (OR) with $95 \%$ confidence interval $(\mathrm{Cl})$. Analyses were repeated in a prospective validation cohort $(n=112)$ of hospitalized patients with COVID-19 treated with remdesivir and dexamethasone. Thirty-day mortality in the derivation cohort was $26.2 \%$. In patients who died, the median PTX3 concentration
\end{abstract}

karger@karger.com www.karger.com/jin

Karger!

GOPEN ACCESS
(C) 2022 The Author(s)

Published by S. Karger AG, Basel

This is an Open Access article licensed under the Creative Commons Attribution-NonCommercial-4.0 International License (CC BY-NC) (http://www.karger.com/Services/OpenAccessLicense), applicable to the online version of the article only. Usage and distribution for commercial purposes requires written permission. upon admission was $19.5 \mathrm{ng} / \mathrm{mL}$ (IQR: 12.5-33.3) versus 6.6 $\mathrm{ng} / \mathrm{mL}$ (IQR 2.9-12.3) $(p<0.0001)$ for survivors. After adjustment for covariates, the odds of 30-day mortality increased two-fold for each doubling of PTX3 (OR 2.03 [95\% Cl: 1.233.34], $p=0.006)$, which was also observed in the validation cohort (OR 1.70 [95\% Cl: 1.09-2.67], $p=0.02$ ). Similarly, PTX3 levels were associated with MV. After adjustment for covariates, OR of MV was 2.34 (95\% Cl: 1.33-4.12, $p=0.003$ ) in the derivation cohort and 1.64 (95\% Cl: $1.03-2.62, p=0.04)$ in the validation cohort. PTX3 appears to be a useful clinical biomarker to predict 30-day respiratory failure and mortality risk in COVID-19 patients treated with and without remdesivir and dexamethasone.

(c) 2022 The Author(s). Published by S. Karger AG, Basel

\section{Introduction}

The coronavirus disease 2019 (COVID-19) pandemic caused by severe acute respiratory syndrome coronavirus-2 (SARS-CoV-2) has put unprecedented pressure on societies and hospital resources worldwide. The disease 
course is unpredictable, ranging from asymptomatic over mild symptoms to respiratory failure and death within days to weeks [1-3]. Clinical markers of progression are urgently needed to stratify patients at symptom onset to guide treatment strategies before the development of severe disease. Thus, a biomarker or a combination of biomarkers that can predict the disease progression may be beneficial.

Several biomarkers have been associated with severe illness and mortality in patients with COVID-19. Among others, these include increased white blood cell count, decreased lymphocyte and platelet counts, elevated plasma levels of creatinine kinase, alanine aminotransferase, lactate dehydrogenase, C-reactive protein (CRP), ferritin, interleukin-6 (IL-6), and D-dimer, and signs of complement aberrations [4-8].

The long pentraxin PTX3 has been suggested as a possible biomarker in COVID-19 $[9,10]$. CRP and PTX3 belong to the pentraxin protein superfamily characterized by the presence of a pentraxin domain in their C-terminal region that interacts with several ligands, including extracellular matrix components and complement molecules as well as a range of pathogens [11]. CRP is predominantly produced in the liver in response to IL-6 [12]. In contrast, PTX3 is concomitantly expressed in the myeloid lineage, fibroblasts, and vascular cells in response to proinflammatory signals such as tumor necrosis factor-alpha, interleukin-1, and toll-like receptor engagement [13]. CRP and PTX3 have been shown to be useful serological inflammatory biomarkers in, e.g., cardiovascular disease, rheumatoid arthritis, and sepsis $[14,15]$. While CRP may have a delayed response, PTX3 induces a rapid response upon stimulation that reflects in situ tissue inflammation and damage $[12,16]$.

While it has been documented that PTX3 is important for different biological processes, it is unknown whether the PTX3 response observed under acute inflammatory conditions is indeed beneficial or harmful or both for the host [17]. Based on its biological role, tissue distribution, and usefulness as a biological outcome marker in critically ill septic patients, we hypothesized that PTX3 might be a valuable prognostic biomarker for hospitalized COVID-19 patients.

\section{Materials and Methods}

\section{Study Population}

The derivation study included adults aged 18 years or older admitted to Copenhagen University Hospital - Amager and Hvidovre Hospital with confirmed SARS-CoV-2 infection between
March 10 and May 31, 2020. Details of the cohort have been described previously [18]. In brief, consecutive cases admitted were included. All cases were confirmed by reverse transcription-polymerase chain reaction on an oropharyngeal swab or lower respiratory tract specimen. Data including patient characteristics, vital parameters, and laboratory measurements were extracted from electronic health records and managed using Research Electronic Data Capture browser-based software (REDCap; Vanderbilt, Nashville, TN, USA). The validation cohort included individuals admitted to Copenhagen University Hospital - Amager and Hvidovre Hospital between September 7 and December 14, 2020, who were treated with remdesivir and dexamethasone. Data collected for this cohort were limited to demographics, comorbidity, and outcome [19]. A blood sample was drawn within 4 days of admission and for some individuals during follow-up. If more than one sample was drawn from the same patient, the first sample was used in the primary analysis. Samples were separated by centrifugation and stored at $-80^{\circ} \mathrm{C}$.

\section{Standard Biochemical Analyses}

CRP, ferritin, ALAT, LDH, creatinine, urea, and blood leukocyte count were taken routinely in the derivation cohort.

\section{Luminex-Based IL-6 Analysis}

Serum IL-6 levels were determined according to the manufacturer's instructions using magnetic fluorescently labeled microsphere beads (R\&D Systems, Abingdon, UK) and analyzed on a BioPlex 200 (Bio-Rad) in the derivation cohort.

\section{Enzyme-Linked Immunosorbent Assay-Based PTX3 Analysis}

Plasma PTX3 levels were quantified in a specific sandwich enzyme-linked immunosorbent assay developed in the Laboratory of Molecular Medicine, Rigshospitalet, according to previously described procedures [20]. The assay was optimized for automated analysis in a 384-well format on the Biomek FX robotic system (Beckman Coulter, Fullerton, CA, USA) [21].

\section{Statistical Analyses}

PTX3 was used as a continuous variable or categorical variable in tertiles. PTX3, CRP, IL-6, lymphocyte count, and urea values were $\log$-transformed $(\log 2)$ in the analyses and backtransformed when presented. Baseline characteristics were reported as frequencies with percentages or medians with interquartile range (IQR). Comparison between COVID-19 patient groups was performed using $\chi^{2}$ test, Fisher's exact test, or MannWhitney $U$ test, as appropriate. Kaplan-Meier survival plots were drawn for each of the three categories and compared by the logrank test. Odds ratios (ORs) with 95\% confidence intervals (CIs) were estimated using logistic regression analysis. Receiver-operating characteristic curve (ROC) analysis was performed to evaluate mortality discrimination. To plot sequential values of PTX3, a B-spline with knots at the quantiles and the smoothing parameter set to zero was constructed and compared by Monte Carlo simulation. A $p$ value $<0.05$ was considered significant. Data analysis was performed using $\mathrm{R}$ software version 4.0.2 (R Foundation for Statistical Computing, Vienna, Austria) and IBM SPSS version 27. 
Table 1. Baseline characteristics, laboratory values, and clinical presentation of the derivation cohort

\begin{tabular}{llllc}
\hline Variables & All $(n=126)$ & Survivors $(n=92)$ & Nonsurvivors $(n=34)$ & $p$ value \\
\hline Age, years & $72[58,81]$ & $65[53,77]$ & $81[72,87]$ & $<0.0001$ \\
Female & $73(58.4)$ & $54(58.7)$ & $19(57.6)$ & 0.91 \\
Comorbidity & $126(99.2)$ & $91(98.9)$ & $34(100)$ & 1.0 \\
$\quad$ Hypertension & $60(47.6)$ & $36(38.7)$ & $24(72.7)$ & $<0.001$ \\
Diabetes & $40(31.7)$ & $26(28.0)$ & $14(42.4)$ & 0.13 \\
Cardiovascular disease & $71(56.3)$ & $45(48.4)$ & $26(78.8)$ & 0.002 \\
Chronic lung disease & $13(10.3)$ & $9(9.7)$ & $3(9.1)$ & 0.92 \\
Malignancy & $19(15.1)$ & $13(14.0)$ & $6(18.2)$ & 0.56 \\
Others & $68(54.0)$ & $48(51.6)$ & $20(60.6)$ & 0.37 \\
Biochemistry & & & & $<0.0001$ \\
P-PTX3, ng/mL & $8.6[4.1,18.3]$ & $6.6[2.9,12.3]$ & $19.5[12.5,35.3]$ & 0.18 \\
P-CRP, mg/L & $98[49,159]$ & $97[49,151]$ & $126[48,217]$ & 0.14 \\
P-ferritin, $\mu g / L$ & $621[277,1,255]$ & $544[264,1,146]$ & $882[338,1,581]$ & $<0.0001$ \\
S-IL-6, pg/mL & $77.0[36,170]$ & $59[29,95]$ & $195[117,356]$ & 0.05 \\
B-lymphocyte, $\times 10^{9}$ IL & $1.0[0.7,1.3]$ & $1.0[0.8,1.3]$ & $0.8[0.6,1.2]$ & 0.10 \\
P-LDH, units/L & $333[252,444]$ & $325[238,417]$ & $381[268,459]$ & 0.13 \\
P-ALAT, units/L & $32(23-56)$ & $32(21-56)$ & $34[28,59]$ & $<0.0001$ \\
P-creatinine, $\mu$ mol/L & $91[75,112]$ & $83[69,103]$ & $111[94,150]$ & $<0.0001$ \\
P-urea, mmol/L & $6.6[4.3,9.9]$ & $5.7[4.0,8.0]$ & $9.2[8.1,13.3]$ & 0.05 \\
Clinical presentation & & & & 0.004 \\
Respiratory rate/min & $20[18,28]$ & $20[18,26]$ & $23[20,30]$ & 0.83 \\
Oxygen saturation, \% & $95[93,97]$ & $96[94,98]$ & $94[91,96]$ & 0.06 \\
Temperature, ${ }^{\circ} \mathrm{C}$ & $37.5[37.0,38.0]$ & $37.0[36.0,39.0]$ & $38.0[37.0,38.0]$ & 0.71 \\
Pulmonary infiltration & $105(83.3)$ & $74(79.6)$ & $31(93.9)$ & $7[5,14]$ \\
Days with symptoms & $7[5,10]$ & $7[5,10]$ & & \\
\hline
\end{tabular}

Values denote median [interquartile range] or number (\%). CRP, C-reactive protein; LDH, lactate dehydrogenase; IL-6, interleukin-6; ALAT, alanine aminotransferase.

\section{Results}

\section{Derivation Cohort}

From March 10 to May 31, 2020, 332 patients with verified COVID-19 were admitted. Of these, 126 had a plasma and serum sample available for analysis. Baseline characteristics are shown in Table 1. Thirty-three of 126 patients died within 30 days (26.2\%). Nonsurvivors were older, more often had hypertension or other cardiovascular disease, had higher plasma levels of IL-6, creatinine, and urea, and had lower blood lymphocyte count and lower peripheral oxygen saturation. Sex, body mass index, diabetes mellitus, chronic lung disease, plasma levels of CRP, ferritin, ALAT, and LDH, blood leukocyte count, respiratory rate, and duration of symptoms prior to admission did not differ significantly between survivors and nonsurvivors $(p>0.05)$.

Baseline plasma PTX3 levels upon admission to the hospital were significantly higher in nonsurvivors compared to survivors (19.5 vs. $6.6 \mathrm{ng} / \mathrm{mL}, p<0.0001)$. Indi- viduals in the highest tertile $(>13.9 \mathrm{ng} / \mathrm{mL})$ had a 30 -day mortality of $54.8 \%$ compared to $19.1 \%$ and $4.8 \%$ for values between 5.8 and $13.9 \mathrm{ng} / \mathrm{mL}$, or $<5.8 \mathrm{ng} / \mathrm{mL}$ (log-rank test: $p<0.0001$ ) (Fig. 1a).

The unadjusted OR of 30-day mortality was 2.61 (95\% CI: 1.73-3.93, $p<0.0001$ ) per doubling of PTX3 level. After adjustment for age, hypertension, cardiovascular disease, oxygen saturation, blood lymphocyte count, and plasma levels of ferritin, urea, and IL-6, the OR was 2.03 (95\% CI: 1.23-3.34, $p=0.006$ ) (Table 2).

Two-hundred and eight plasma samples were available during follow-up for 177 patients (Fig. 2), i.e., samples collected more than 4 days after admission contributed. Among patients who died, PTX3 levels were high at baseline and increased over the first week. For patients who survived, PTX3 levels were lower and declined over time. The differences were statistically significant (day 7 : $p=0.0008)$.

Twenty patients required MV (15.9\%). The unadjusted OR of MV was 1.77 (95\% CI: $1.24-2.51, p=0.002)$ per 


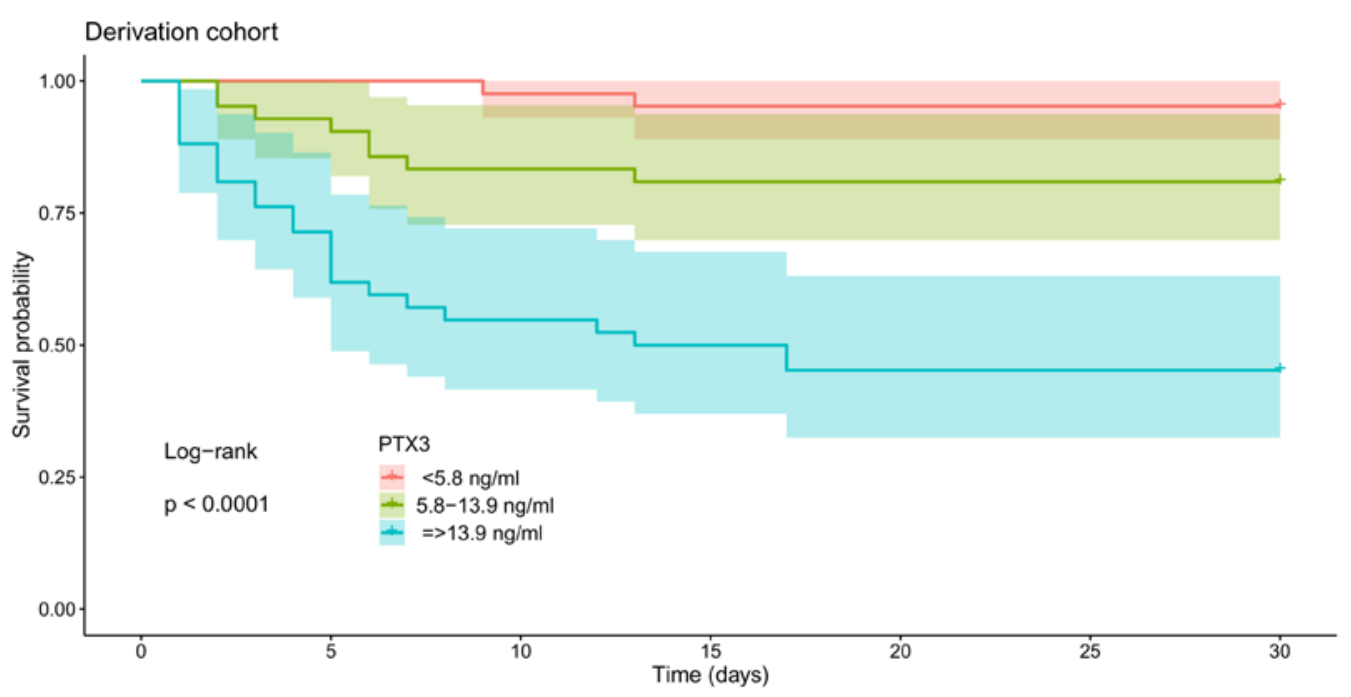

a \begin{tabular}{lllllll}
\multicolumn{2}{l}{ Number at risk } \\
42 & 42 & 41 & 40 & 40 & 40 & 40 \\
42 & 39 & 35 & 34 & 34 & 34 & 34 \\
42 & 30 & 23 & 21 & 19 & 19 & 19 \\
\hline 0 & 5 & 10 & 15 & 20 & 25 & 30
\end{tabular}

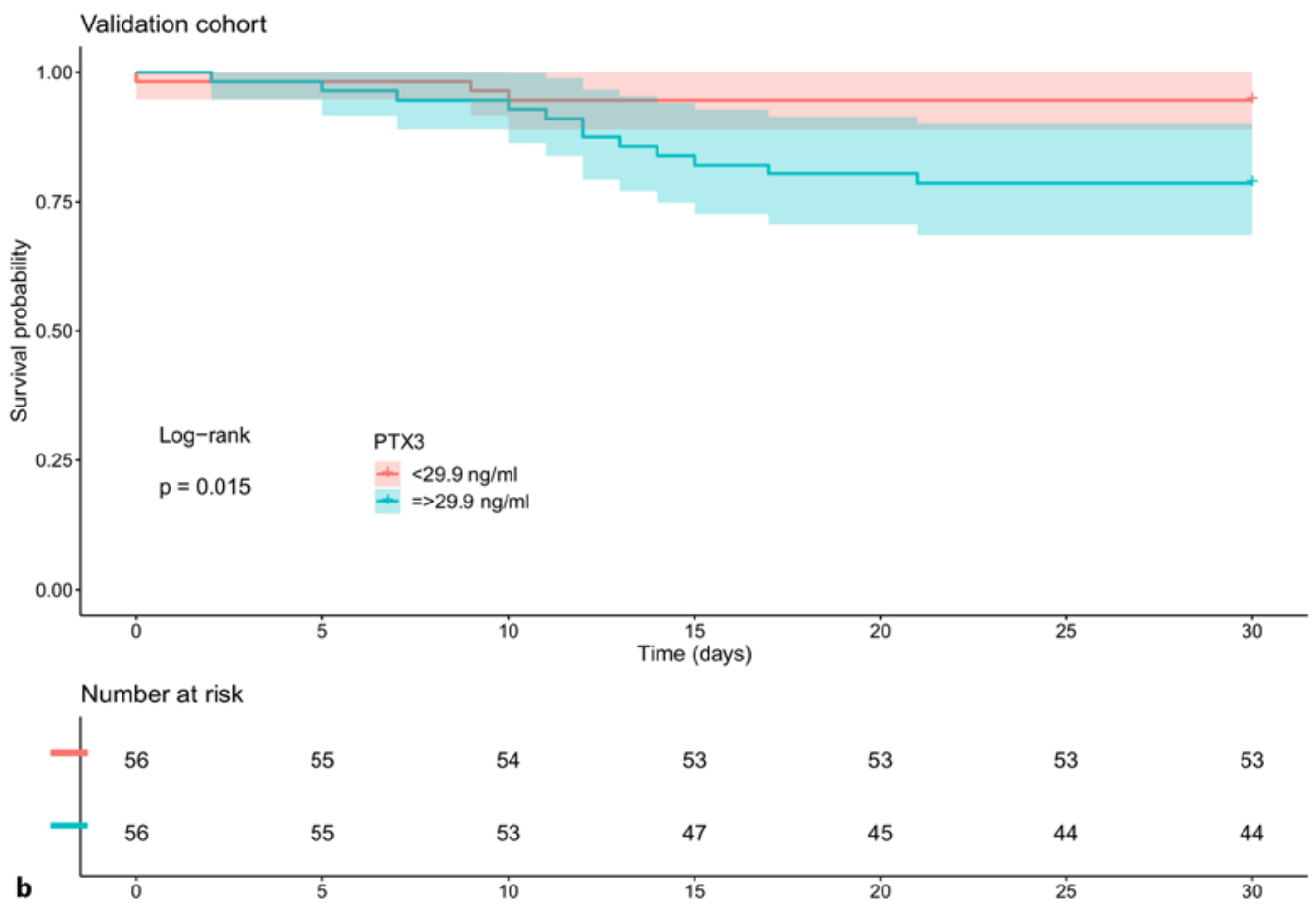

Fig. 1. Thirty-day survival from COVID-19 by plasma level of long pentraxin-3. a Kaplan-Meier curves depicting 30-day survival in the derivation cohort $(n=126)$ according to plasma PTX3 level at baseline divided into tertiles; $<5.8 \mathrm{ng} / \mathrm{mL}$ (red), $5.8-13.9 \mathrm{ng} / \mathrm{mL}$ (green), and $\geq 13.9 \mathrm{ng} / \mathrm{mL}$ (blue), with $95 \%$ confidence interval.
Log-rank test: $p<0.0001$. b Kaplan-Meier curves depicting 30-day survival in the validation cohort $(n=112)$ according to plasma PTX3 level at baseline (start of remdesivir and dexamethasone) divided by the median; $<29.9 \mathrm{ng} / \mathrm{mL}$ (red) and $\geq 29.9 \mathrm{ng} / \mathrm{mL}$ (blue), with $95 \%$ confidence interval. Log-rank test: $p<0.015$. 
Table 2. Logistic regression analysis of factors associated with 30-day mortality in the derivation and validation cohorts

\begin{tabular}{llll}
\hline & \multicolumn{2}{l}{ Derivation cohort $(n=126)$} & \\
\cline { 2 - 4 } & \multicolumn{1}{l}{ crude OR $(95 \% \mathrm{Cl})$} & adjusted OR $(95 \% \mathrm{Cl})^{1}$ & $p$ value \\
\hline PTX3, per doubling & $2.61(1.73-3.93)$ & $2.03(1.23-3.34)$ & 0.006 \\
Age, per year increment & $1.07(1.03-1.11)$ & $1.05(0.98-1.13)$ & 0.15 \\
Hypertension & $4.22(1.77-10.10)$ & $3.21(0.41-24.82)$ & 0.27 \\
Cardiovascular disease & $3.96(1.57-10.03)$ & $2.68(0.21-33.85)$ & 0.45 \\
P-CRP, per doubling & $1.29(0.90-1.86)$ & $0.96(0.50-1.83)$ & 0.90 \\
P-ferritin, per doubling & $1.21(0.93-1.56)$ & $0.92(0.57-1.49)$ & 0.73 \\
S-IL-6, per doubling & $2.32(1.59-3.39)$ & $2.05(1.07-3.93)$ & 0.03 \\
B-lymphocyte count, per doubling & $0.47(0.22-0.97)$ & $0.62(0.21-1.79)$ & 0.37 \\
P-urea, per doubling & $4.25(2.06-8.76)$ & $1.54(0.50-4.72)$ & 0.45 \\
Oxygen saturation, per \% increment & $0.85(0.75-0.96)$ & $1.00(0.80-1.25)$ & 0.98 \\
\hline & Validation cohort $(n=112)$ & & $p$ value \\
\hline PTX3, per doubling & crude OR (95\% Cl) & adjusted OR (95\% CI) & 0.02 \\
Age, per year increment & $1.78(1.15-2.76)$ & $1.70(1.09-2.67)$ & 0.02 \\
\hline
\end{tabular}

OR, odds ratio; $\mathrm{Cl}$, confidence interval; CRP, C-reactive protein; IL-6, interleukin-6. ${ }^{1}$ Adjusted for age, hypertension, cardiovascular disease, oxygen saturation, blood lymphocyte count, and plasma levels of ferritin, urea, and IL-6. ${ }^{2}$ Adjusted for age.

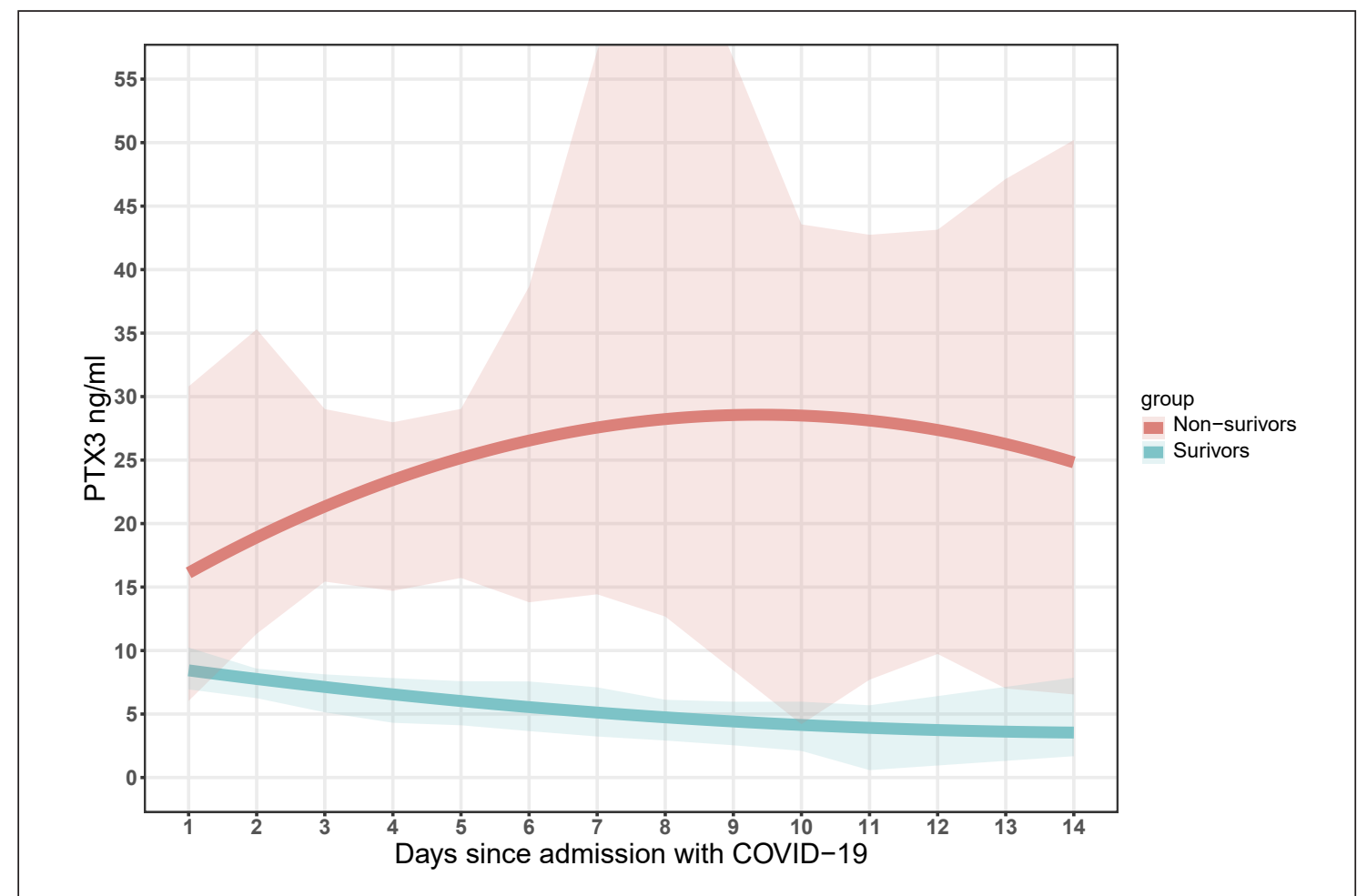

Fig. 2. Levels of PTX3 over time for COVID-19 patient survivors and nonsurvivors. Time-dependent PTX3 levels with $95 \%$ confidence interval in 30-day survivors (blue) and nonsurvivors (red), 208 samples $(n=177)$. The differences were statistically significant (day $7: p=0.0008)$. 
Table 3. Baseline characteristics, laboratory values, and clinical presentation of the validation cohort

\begin{tabular}{lllll}
\hline Variables & All $(n=112)$ & Survivors $(n=96)$ & Nonsurvivors $(n=16)$ & $p$ value \\
\hline Age, years & $62[51-73]$ & $59[49-72]$ & $74[66-78]$ & 0.003 \\
$\begin{array}{l}\text { Female } \\
\text { Comorbidity, no }\end{array}$ & $37(33.0)$ & $32(33.0)$ & $5(33.3)$ & 0.98 \\
$\begin{array}{l}\text { Biochemistry } \\
\quad \text { P-PTX3, ng/mL }\end{array}$ & $96(85.7)$ & $82(84.5)$ & $14(93.3)$ & 0.37 \\
$\begin{array}{l}\text { Clinical presentation } \\
\quad \text { Pulmonary infiltration }\end{array}$ & $29.9[13.7-56.1]$ & $26.5[12.2-51.0]$ & $56.5[30.9 .4-96.4]$ & 0.008 \\
$\quad$ Days with symptoms & $107(95.5)$ & $93(95.9)$ & $14(93.3)$ & 0.66 \\
\hline
\end{tabular}

Values denote median [interquartile range] or number (\%).

doubling of PTX3 level. After adjustment for age, hypertension, cardiovascular disease, oxygen saturation, blood lymphocyte count, and plasma levels of ferritin, urea, and IL-6, the OR was 2.34 (95\% CI: 1.33-4.12, $p=0.003$ ).

To evaluate the discrimination of mortality using PTX3 compared to other predictors of 30-day mortality, ROC curve analysis was performed. The AUC for PTX3 and IL-6 was comparable (0.84 [95\% CI: $0.75-0.93$ ] vs. 0.79 [95\% CI: $0.69-0.90], p=0.39)$, but better than CRP (0.84 [95\% CI: $0.75-0.93$ ] vs. 0.56 [95\%: 0.43-0.69], $p<$ 0.001 ) (online suppl. Fig. 1; for all online suppl. material, see www.karger.com/doi/10.1159/000521612).

\section{Validation Cohort}

From September 7 to December 14, 2020, 175 patients with verified COVID-19 received treatment with remdesivir and dexamethasone for moderate to severe COVID-19. Of these, 112 patients had a plasma sample within 3 days of start of remdesivir and dexamethasone and were included in this study. Fifteen patients died within 30 days (13.4\%). Thirteen progressed to respiratory failure and required MV (11.6\%).

The median age in the cohort was 62 years (IQR 5173 ) and 37 (33\%) were females. There was a significant difference between the age of survivors (59 years, IQR $49-72$ ) and nonsurvivors (74 years, IQR 66-78) ( $p=$ 0.003 ). Baseline characteristics of the entire cohort and the subgroup survivors and nonsurvivors according to 30-day mortality are outlined in Table 3.

Baseline plasma levels of PTX3 were significantly higher in nonsurvivors compared to survivors (median 56.5 vs. $26.5 \mathrm{ng} / \mathrm{mL}, p=0.008$ ). Individuals with a PTX3 level above the median $(\geq 29.9 \mathrm{ng} / \mathrm{mL})$ had a 30 -day mortality of $21.4 \%$ compared to $5.4 \%$ for individuals below the median (log-rank test: $p<0.015$ ) (Fig. 1b). By uni- variate analysis, each doubling of PTX3 was associated with an OR of 1.78 (95\% CI: 1.15-2.76) for 30-day mortality (Table 2). After adjustment for the only other factor associated with 30-day mortality (age), the OR was 1.70 (95\% CI: 1.09-2.67, $p=0.02$ ). Thirteen individuals required MV. By univariate analysis, each doubling of PTX3 was associated with an OR of 1.64 (95\% CI: 1.06-2.55) for MV. No other factor was associated with MV in the validation cohort.

\section{Discussion}

It is crucial to acquire tools to evaluate and prioritize the high-risk patients in the initial stages of COVID-19 disease progression to help predict and possibly alter the disease trajectory. Several prognostic biomarkers and prediction models, e.g., using machine learning, have been established throughout the COVID-19 pandemic, illustrating the complexity and urgent need for understanding SARS-CoV-2 infection progression [22-25]. PTX3 is barely measurable in healthy subjects with a concentration $\leq 2 \mathrm{ng} / \mathrm{mL}$ [26] and has previously been shown to increase rapidly (peak at 6-8 h) during sepsis and other inflammatory conditions $[14,16]$. This observation suggests that PTX3 may be an advantageous early predictor of disease severity in critically ill patients. In this study, we set out to evaluate PTX3 as a prognostic biomarker for short-term mortality in hospitalized COVID-19 patients.

In the present study, we show that PTX3 is a significant and robust marker of mortality in COVID-19 patients. The findings were substantiated in two independent cohorts from the first COVID-19 wave in the spring 2020 and the second wave in autumn 2020. PTX3 was independent of established COVID-19 clinical markers such as 
sex, age, and comorbidities in both cohorts. There were several differences between the cohorts which emphasized the robustness of our findings. In the derivation cohort (from the first wave of COVID-19 in Denmark), the overall mortality was $26.2 \%$, whereas the mortality in the validation cohort (from the second wave of COVID-19 in Denmark) was $13.4 \%$. Furthermore, the median age in the derivation cohort compared to the validation cohort was 72 years and 62 years, respectively. The gender distribution also differed considerably between the cohorts, with the percentage of female patients in the derivation cohort constituting $58.4 \%$, while only $33 \%$ were female in the validation cohort. First and foremost, the treatment regimen differed notably between the two cohorts, with only patients in the validation cohort receiving treatment with remdesivir and dexamethasone for moderate to severe COVID-19. We observed that the median level of PTX3 was nearly 3.5 times elevated in the validation cohort compared to the derivation cohort, $29.9 \mathrm{ng} / \mathrm{mL}$ and $8.6 \mathrm{ng} / \mathrm{mL}$, respectively. This is compatible with studies showing that glucocorticoid administration increases systemic PTX3 levels $[27,28]$, while other acute-phase reactants may be downregulated. The expression of PTX3 is influenced by corticosteroids in a cell compartment-specific manner since glucocorticosteroids enhance PTX3 expression in fibroblasts and endothelial cells, while it is downregulated by glucocorticosteroids in myeloid cells [29]. Previously, we have shown that in patients given methylprednisolone undergoing total knee arthroplasty, the level of PTX3 increases rapidly, and the difference between the treatment group and placebo group was in the same magnitude as seen in the present study $(4.7 \mathrm{ng} / \mathrm{mL}$ vs. $14.6 \mathrm{ng} / \mathrm{mL}$ after 6 h) [28]. Despite the complex regulation of the PTX3 gene, PTX3 was a strong predictor in both cohorts, albeit somewhat weaker in the second cohort.

A smooth plot based on patients from the derivation cohort revealed that PTX3's signal to noise ratio between survivors and nonsurvivors continued to increase up to around 1 week after admission, which also emphasizes the usefulness and strength of PTX3 as a prognostic parameter in COVID-19 patients. Our study is compatible with other recent studies showing PTX3 to be associated with disease severity and mortality in COVID-19 patients $[9,10,30]$. However, in these studies, implementation of dexamethasone was not taken into account. Thus, our study provides particular insight into the influence of dexamethasone on PTX3 levels and illustrates that cutoff levels might be chosen with respect to the treatment given, if PTX3 should be implemented in prognostic algorithms.
PTX3 is not only a biomarker but an active modulator and driver of the innate inflammatory response [16]. In a recent study by Divella et al. [31], they suggest that PTX3 plays a central role in mediating kidney damage in ischemia-reperfusion (I/R) injury. Thus, based on our findings, it may be pertinent to suggest that pharmacological inhibition of PTX3 could be beneficial. However, Lech et al. [32] propose the opposite scenario and demonstrate that lack of PTX3 aggravates renal I/R injury in Ptx3-deficient mice. These apparently contradictory results may be due to the fact that different animal and model systems were used. Nevertheless, complete lack of PTX3 may be undesired and associated with disease as will high levels seen in systemic uncontrolled inflammatory reactions.

In the derivation cohort, we had the chance to measure IL- 6 and CRP, both have been demonstrated to be potential biomarkers of severe outcome and death in hospitalized COVID- 19 patients $[5,9]$. In our study, both PTX3 and IL- 6 appeared to perform better than CRP. However, PTX3 in regression models was independent of IL-6, indicating these two parameters reflect different pathways in the COVID-19 pathophysiology and could both contribute to prognostic accuracy if combined.

Some limitations need to be addressed regarding the present study. As with all observational studies, the present work might be subject to potential bias due to unknown confounders, and we cannot draw definitive conclusions regarding causality. As addressed above, the derivation and validation cohorts differ notably regarding age distribution, sex distribution, availability of covariates, and pharmacological treatment of the patients.

In conclusion, in the present study, we show that PTX3 is an independent marker of 30-day mortality and respiratory failure in hospitalized COVID-19 patients in two independent cohorts with different treatment regimes. The findings imply that PTX3 may represent an early and cost-efficient tool in patient triage and management.

\section{Acknowledgments}

We thank Mss Bettina Eide Holm, Jytte Bryde Clausen, and Anna Louise Sørensen for excellent technical assistance. We would also like to thank the patients and staff at Copenhagen University Hospital - Amager and Hvidovre Hospital, from where these data originated. 


\section{Statement of Ethics}

The study was approved by the Danish Patient Safety Authority (Record No. 31-1521-309) and the Regional Data Protection Center (Record No. P-2020-492). The ethics committee approved measurements of biomarkers in stored samples from the biobank of the Capital Region of Denmark (Record No. H-20047597). The committee exempted a requirement of individual informed consent.

\section{Conflict of Interest Statement}

The authors have no conflicts of interest to declare.

\section{Funding Sources}

This work was supported by grants from the Carlsberg Foundation (CF20-0045), the Novo Nordisk Foundation (NFF205A0063505), and in part by Holbaek Hospital's Research
Foundation, and R\&D Systems provided a discount on the reagents used in this study (IL-6 analysis).

\section{Author Contributions}

C.B.H., P.G., and T.B. performed conceptualization and study design. C.B.H., M.E.E.M., L.P.-A., L.P., and S.B.I. performed the data collection. C.B.H., H.S., P.G., and T.B. performed the data analysis. C.B.H., P.G., and T.B. wrote the first draft of the manuscript. All authors read and approved the final manuscript.

\section{Data Availability Statement}

Data are available from the corresponding author on reasonable request.

\section{References}

1 Huang C, Wang Y, Li X, Ren L, Zhao J, Hu Y, et al. Clinical features of patients infected with 2019 novel coronavirus in Wuhan, China. Lancet. 2020 Feb 15;395(10223):497-506.

$2 \mathrm{Wu}$ Z, McGoogan JM. Characteristics of and important lessons from the Coronavirus Disease 2019 (COVID-19) outbreak in China: summary of a report of 72,314 cases from the Chinese Center for Disease Control and Prevention. JAMA. 2020 Apr 7;323(13):1239-42.

3 Oran DP, Topol EJ. The proportion of SARSCoV-2 infections that are asymptomatic: a systematic review. Ann Intern Med. 2021 May;174(5):655-62.

4 Danwang C, Endomba FT, Nkeck JR, Wouna DLA, Robert A, Noubiap JJ. A meta-analysis of potential biomarkers associated with severity of coronavirus disease 2019 (COVID-19). Biomark Res. 2020;8:37.

5 Del Valle DM, Kim-Schulze S, Huang HH, Beckmann ND, Nirenberg S, Wang B, et al. An inflammatory cytokine signature predicts COVID-19 severity and survival. Nat Med. 2020 Oct;26(10):1636-43.

6 Liao D, Zhou F, Luo L, Xu M, Wang H, Xia J, et al. Haematological characteristics and risk factors in the classification and prognosis evaluation of COVID-19: a retrospective cohort study. Lancet Haematol. 2020 Sep;7(9):e671-8.

7 Shen B, Yi X, Sun Y, Bi X, Du J, Zhang C, et al. Proteomic and metabolomic characterization of COVID-19 patient sera. Cell.. 2020 Jul 9;182(1):59-72.e15.

8 Shi J, Li Y, Zhou X, Zhang Q, Ye X, Wu Z, et al. Lactate dehydrogenase and susceptibility to deterioration of mild COVID-19 patients: a multicenter nested case-control study. BMC Med. 2020 Jun 3;18(1):168.
9 Brunetta E, Folci M, Bottazzi B, De Santis M, Gritti G, Protti A, et al. Macrophage expression and prognostic significance of the long pentraxin PTX3 in COVID-19. Nat Immunol. 2020 Nov 18;22(1):19-24.

10 Gutmann C, Takov K, Burnap SA, Singh B, Ali $\mathrm{H}$, Theofilatos $\mathrm{K}$, et al. SARS-CoV-2 RNAemia and proteomic trajectories inform prognostication in COVID-19 patients admitted to intensive care. Nat Commun. 2021 Jun 7;12(1):3406.

11 Garlanda C, Bottazzi B, Magrini E, Inforzato A, Mantovani A. PTX3, a humoral pattern recognition molecule, in innate immunity, tissue repair, and cancer. Physiol Rev. 2018 Apr 1;98(2):623-39.

12 Pepys MB, Hirschfield GM. C-reactive protein: a critical update. J Clin Invest. 2003 Jun; 111(12):1805-12.

13 Bottazzi B, Doni A, Garlanda C, Mantovani A An integrated view of humoral innate immunity: pentraxins as a paradigm. Annu Rev Immunol. 2010;28(1):157-83.

14 Muller B, Peri G, Doni A, Torri V, Landmann $\mathrm{R}$, Bottazzi B, et al. Circulating levels of the long pentraxin PTX3 correlate with severity of infection in critically ill patients. Crit Care Med. 2001 Jul;29(7):1404-7.

15 Sproston NR, Ashworth JJ. Role of C-reactive protein at sites of inflammation and infection. Front Immunol. 2018;9:754.

16 Garlanda C, Bottazzi B, Bastone A, Mantovani A. Pentraxins at the crossroads between innate immunity, inflammation, matrix deposition, and female fertility. Annu Rev Immunol. 2005;23:337-66.
17 Magrini E, Mantovani A, Garlanda C. The dual complexity of PTX3 in health and disease: a balancing act? Trends Mol Med. 2016 Jun;22(6):497-510.

18 Israelsen SB, Kristiansen KT, Hindsberger B, Ulrik CS, Andersen O, Jensen M, et al. Characteristics of patients with COVID-19 pneumonia at Hvidovre Hospital, March-April 2020. Dan Med J. 2020 May 15;67(6):A05200313.

19 Benfield T, Bodilsen J, Brieghel C, Harboe ZB, Helleberg M, Holm C, et al. Improved survival among hospitalized patients with $\mathrm{CO}$ VID-19 treated with remdesivir and dexamethasone. A nationwide population-based cohort study. Clin Infect Dis. 2021 Dec 6; 73(11):2031-6.

20 Bastrup-Birk S, Skjoedt MO, Munthe-Fog L, Strom JJ, Ma YJ, Garred P. Pentraxin-3 serum levels are associated with disease severity and mortality in patients with systemic inflammatory response syndrome. PLoS One. 2013; 8(9):e73119.

21 Vengen IT, Enger TB, Videm V, Garred P. Pentraxin 3, ficolin-2 and lectin pathway associated serine protease MASP-3 as early predictors of myocardial infarction: the HUNT2 study. Sci Rep. 2017;7(7):43045.

22 Laing AG, Lorenc A, Del Molino Del Barrio I, Das A, Fish M, Monin L, et al. A dynamic COVID-19 immune signature includes associations with poor prognosis. Nat Med. 2020 Oct;26(10):1623-35.

23 Mathew D, Giles JR, Baxter AE, Oldridge DA, Greenplate AR, Wu JE, et al. Deep immune profiling of COVID-19 patients reveals distinct immunotypes with therapeutic implications. Science. 2020 Sep 4;369(6508):eabc8511. 
24 Antunez Muiños PJ, López Otero D, AmatSantos IJ, López País J, Aparisi A, Cacho Antonio CE, et al. The COVID-19 lab score: an accurate dynamic tool to predict in-hospital outcomes in COVID-19 patients. Sci Rep. 2021 Apr 30;11(1):9361.

25 Jimenez-Solem E, Petersen TS, Hansen C, Hansen C, Lioma C, Igel C, et al. Developing and validating COVID-19 adverse outcome risk prediction models from a bi-national $\mathrm{Eu}$ ropean cohort of 5594 patients. Sci Rep. 2021 Feb 5;11(1):3246.

26 Yamasaki K, Kurimura M, Kasai T, Sagara M, Kodama T, Inoue K. Determination of physiological plasma pentraxin 3 (PTX3) levels in healthy populations. Clin Chem Lab Med. 2009;47(4):471-7.
27 Lerzo F, Peri G, Doni A, Bocca P, Morandi F, Pistorio A, et al. Dexamethasone prophylaxis in pediatric open heart surgery is associated with increased blood long pentraxin PTX3: potential clinical implications. Clin Dev Immunol. 2011;2011:730828.

28 Lindberg-Larsen V, Kehlet H, Pilely K, Bagger J, Rovsing ML, Garred P. Preoperative methylprednisolone increases plasma Pentraxin 3 early after total knee arthroplasty: a randomized, double-blind, placebo-controlled trial. Clin Exp Immunol. 2018 Mar;191(3):356-62.

29 Doni A, Mantovani G, Porta C, Tuckermann J, Reichardt HM, Kleiman A, et al. Cell-specific regulation of PTX3 by glucocorticoid hormones in hematopoietic and nonhematopoietic cells. J Biol Chem. 2008 Oct 31; 283(44):29983-92.
30 Schirinzi A, Pesce F, Laterza R, D’Alise MG, Lovero R, Fontana A, et al. Pentraxin 3: potential prognostic role in SARS-CoV-2 patients admitted to the emergency department. J Infect. 2021 Apr;82(4):84-123.

31 Divella C, Stasi A, Franzin R, Rossini M, Pontrelli P, Sallustio F, et al. Pentraxin-3-mediated complement activation in a swine model of renal ischemia/reperfusion injury. Aging. 2021 Apr 20;13(8):10920-33.

32 Lech M, Römmele C, Gröbmayr R, Eka Susanti H, Kulkarni OP, Wang S, et al. Endogenous and exogenous pentraxin-3 limits postischemic acute and chronic kidney injury. Kidney Int. 2013 Apr;83(4):647-61. 